\title{
Frecuencia y punto de vista: procedimientos narrativos para estructurar el relato
}

\section{Frequency and point of view: narrative procedures to structure the story}

\author{
José Prósper Ribes
}

Profesor titular, Universidad Politécnica de Valencia

Fecha de recepción: 26 de marzo de 2012

Fecha de revisión: 15 de enero de 2012

Para citar este artículo: Prósper Ribes, J. (2013): Frecuencia y punto de vista: procedimientos narrativos para estructurar el relato, Icono 14, volumen 11 (1), pp. 281-302. doi: 10.7195/ri14.v11i1.192 


\section{Resumen}

La utilización combinada de la frecuencia repetitiva y la variación del punto de vista es un procedimiento narrativo muy adecuado para modificar el sentido de un acontecimiento. En la investigación, abordamos el tema a partir de la definición de los conceptos teóricos fundamentales que sirven de sustento al trabajo. A continuación procedemos al análisis de casos ejemplares en tres películas (El extraño viaje, El hombre que mató a Liberty Valance y Ciudad de Dios) donde en la primera ocasión el acontecimiento se muestra con una evidente restricción informativa que es subsanada en la segunda ocasión en que se muestra de forma que se modifica el sentido final del acontecimiento. Los resultados fundamentales del estudio indican que para controlar el suministro informativo en la primera vez que se muestra el acontecimiento, se suele recurrir a la elipsis y al espacio fuera de campo. A través de la elipsis se elimina un dato fundamental, mientras que el espacio fuera de campo oculta un dato esencial para comprender el acontecimiento.

\section{Palabras clave}

Frecuencia repetitiva - punto de vista - flashback - enigma - elipsis - espacio fuera de campo

\section{Abstract}

The utilization combined of the repetitive frequency and the variation of the point of view is a narrative procedure very appropriate to modify the sense of an event. In the investigation, we approach the topic from the definition of the theoretical fundamental concepts that use as sustenance to the work. Later, we proceed to the analysis of exemplary cases in three movies where in the first occasion the event appears with an evident informative restriction that is corrected in the second occasion in which it appears so that the final sense of the event is modified. The key results of the study indicate that in order to control the information supply, ellipsis and offcamera space are used. The ellipsis eliminates a fundamental information, whereas the off-camera space conceals an essential information to understand the event. 


\section{Key Words}

Repetitive frequency - point of view -flashback - enigma - ellipsis - off-camera space

\section{Introducción}

El factor temporal es esencial a la hora de configurar un relato y su utilización contribuye de manera decisiva a establecer el sentido final de la narración y de sus características discursivas. Es habitual analizar el tiempo a partir de la duración, el orden y la frecuencia. Cuando un acontecimiento que tienen lugar una única vez en la historia se muestra dos o más veces en el discurso, hablamos de frecuencia repetitiva. La utilización de la frecuencia repetitiva junto a la modificación del punto de vista del acontecimiento o situación que se vuelve a mostrar en el relato es un procedimiento narrativo que normalmente parte de la limitación informativa que tiene el espectador de la primera ocasión en que se muestra el suceso para posteriormente completar la información de manera que el espectador tenga un conocimiento integral de lo sucedido.

\section{Objetivos}

1. Exponer el concepto de frecuencia temporal en el relato audiovisual y desarrollar las características de la modalidad de frecuencia repetitiva.

2. Desarrollar el concepto de punto de vista y su utilización en el relato audiovisual.

3. Analizar el uso conjunto de la frecuencia repetitiva y la alternancia del punto de vista para configurar el relato, modificando el sentido de los acontecimientos.

4. Analizar el uso del fuera de campo y de la elipsis como los mecanismos narrativos básicos que permiten establecer una diferencia informativa entre las dos o más ocasiones en que se muestra un acontecimiento.

\section{Metodología}

La metodología ha consistido, como punto de partida, en realizar un estudio so-

DOI: ri14.v11i1.192| ISSN: 1697-8293 | Año 2013 Volumen 11 Nº1 | ICONO14 
bre el estado de la cuestión en la materia a partir de las principales nociones narrativas. Esto nos ha permitido definir los conceptos fundamentales sobre el tiempo y el punto de vista que permiten abordar los mecanismos de restricción informativa para establecer un enigma o una información incompleta sobre un acontecimiento determinado, y su posterior subsanación a través de la repetición de dicho acontecimiento variando el punto de vista. Los criterios de selección de las películas han sido por su relevancia en la utilización de la frecuencia repetitiva y del punto de vista, como recursos narrativos claves para la valoración de los acontecimientos, y la creación de la estructura argumental, independientemente del género, época o nacionalidad. Posteriormente, hemos realizado un análisis exhaustivo a partir del uso de la elipsis y del fuera de campo para restringir la información y la posterior repetición del acontecimiento modificando la posición del punto de vista de forma que se ofrece nueva información relevante para la comprensión del acontecimiento.

\section{Tiempo narrativo y frecuencia}

El tiempo es uno de los elementos claves para establecer el sentido del relato. Para Kohan

La utilización del tiempo en el relato es la manifestación de una visión de ese mundo narrado. Según lo que quieras decir, así lo trabajas. Del trazado lógico y cronológico a las más extremas rupturas de esa linealidad, las variantes son muchas, tantas como relatos existen. (2005, p. 55)

El tiempo narrativo puede ser considerado según diversos criterios siendo el más habitual analizarlo a partir de las relaciones que se establecen entre el tiempo de la historia y el tiempo del discurso. Al respecto, Mario Rajas señala

El tiempo de la historia (TH o tiempo narrado) hace referencia a los parámetros temporales que constituyen el contenido del relato, mientras que el tiempo del discurso (TD o tiempo de la narración) se define como la construcción textual específica de ese tiempo cronométrico en una obra determinada. (2011, p. 360)

Fue Genette quien estableció el estudio del tiempo narrativo a partir de las categorías de orden, duración y frecuencia (1989, p. 90). Hay que tener en cuenta que Genette utiliza los términos tiempo de la historia y tiempo del relato. A través del orden se estudia cómo se disponen dentro del discurso los acontecimientos que

ICONO14 | Año 2013 Volumen 11 № 01 | ISSN: 1697-8293 | DOI: ri14.v11i1.192 
se desarrollan cronológicamente en el universo diegético. La duración establece la relación que existe entre el tiempo que dura un acontecimiento en la historia y el que tiene en el discurso mientras que la frecuencia hace referencia al número de veces que un suceso (o sucesos) es representado en el discurso en relación al número de veces que el suceso (o sucesos) tiene lugar en la historia. Las tres categorías están estrechamente unidas, especialmente el orden y la frecuencia ya que normalmente la frecuencia precisa de un tiempo de referencia que se consigue mediante el establecimiento de una sucesión temporal precisa.

La frecuencia temporal es un concepto hasta cierto punto controvertido ya que se puede considerar que un acontecimiento es siempre único y, por lo tanto, no hay dos acontecimientos idénticos. Para Bal

El fenómeno de la repetición, que está siendo discutido aquí, siempre ha tenido una faceta problemática. Dos acontecimientos no son nunca exactamente iguales. El primer acontecimiento de una serie difiere del que le sigue, aunque sólo sea porque uno es el primero y el otro no. (1990, p. 85)

El propio Genette señala:

La repetición es, en realidad, una construcción mental, que elimina de cada caso todo lo que le pertenece propiamente para conservar sólo lo que comparte con todos los demás de la misma clase, y una abstracción: "el sol", "la mañana", "salir". (1989, p. 172)

Podemos considerar cinco modalidades básicas de frecuencia:

1. Singularidad. Un acontecimiento ocurre una vez en la diégesis y se representa una vez en el discurso (TH1-TD1). Sin duda alguna es la modalidad más utilizada.

2. Frecuencia múltiple. En el discurso se representa $n$ ocasiones lo que en la historia ocurre $\mathrm{x}$ veces (THx-TDn).

3. Frecuencia repetitiva. Un acontecimiento que tiene lugar una única ocasión en la diégesis es mostrado en dos o más ocasiones en el discurso (TH1-TDn).

4. Frecuencia iterativa. En el discurso se muestra en una única vez acontecimientos que tienen lugar en la historia $n$ veces (THn-TDn).

5. Frecuencia cero. Sucesos que se producen en el universo diegético no son mostrados en el discurso. (THn-TD0). Obviamente estamos hablando de la elipsis. De las cinco modalidades de frecuencia, esta última es la que no siempre se considera como un tipo de frecuencia concreta. 
Depende de los autores, podemos encontrar un mayor o menor número de modalidades de frecuencia. Por ejemplo, Bordwell (1999), señala nueve posibilidades o Bal (1990) subdivide la frecuencia múltiple. Sin embargo, las cinco modalidades que señalamos son las más comúnmente aceptadas y engloban todas las variantes posibles.

Sin duda alguna, la frecuencia repetitiva es la menos problemática ya que en esta modalidad el acontecimiento repetido es siempre el mismo y no cabe plantear el inconveniente de la diferencia o disimilitud entre acontecimientos similares pero únicos ni tampoco considerarla como una abstracción. Ahora bien, se considera que su uso debe atender a unos criterios que lo justifiquen. Así para Bal

Nos referiremos a una repetición real cuando un acontecimiento se dé una vez y se presente varias [...] A veces las variaciones de la "perspectiva" se usan también para justificar la necesidad de una repetición, el acontecimiento puede ser el mismo, pero cada actor lo contempla desde su propio punto de vista (1990, p. 86)

Combinar la repetición de un acontecimiento con la modificación del punto de vista para ofrecer una nueva perspectiva de los sucesos es un recurso narrativo que tiene como fundamento cambiar el sentido de los eventos. Para Bordwell esta es una de las justificaciones al uso de la frecuencia repetitiva:

Si la historia se "representa de nuevo", la repetición está sujeta a rigurosas reglas narrativas. Debe motivarse de una manera realista, generalmente a través de la subjetividad de un personaje, como un recuerdo [...] En algunos casos, la dramatización repetida puede justificarse para sacar a la luz información suprimida o ignorada en la primera oportunidad, como en el flashback de Doniphon de El hombre que mató a Liberty Balance (The Man Who Shot Liberty Valance, 1962). Volver a representar el suceso de la historia sin una motivación realista es crear una narración altamente ilimitada, autoconsciente, que sobrepasa las leyes del mundo de la historia y rehace una porción de la acción a voluntad. (1999, p. 80)

El objetivo narrativo muy frecuentemente es o bien plantear un enigma a lo largo de un fragmento del relato y que sea desvelado más adelante a través de la repetición o bien ofrecer una nueva información que modifique la situación de partida y que suele producir un efecto sorpresa en el espectador por lo inesperado de la solución. En todos los casos, es habitual que la repetición del suceso se articule a través del flashback de un narrador, ya sea a través de una declaración a otro personaje o del recuerdo de dicho personaje. Y una de las características básicas

ICONO14 | Año 2013 Volumen 11 № 01 | ISSN: 1697-8293 | DOI: ri14.v11i1.192 
del flashback es que "hace hincapié en el "cómo" ha sucedido algo, más relevante informativamente en estos casos que el "qué" va a suceder" (Rajas, 2011, p. 367). El espectador ya conoce el qué y es frecuentemente el cómo ha sucedido o quién es el responsable donde radica el placer de descubrir el enigma, lo que se convierte en el motor del desarrollo narrativo.

\section{El punto de vista y la presencia del narrador en la frecuencia repetitiva}

Uno de los conceptos más controvertidos en la teoría narrativa, especialmente en la aplicada al relato audiovisual, es el de punto de vista: "Si hay actualmente en la teoría cinematográfica un concepto-paraguas éste es, sin lugar a dudas, el de punto de vista" (Zunzunegui, 1998, p. 187). Efectivamente, el término punto de vista engloba diferentes sentidos Pero es conveniente tener una referencia clara para analizar su aplicación al estudio de las películas que lo utilizan junto a la frecuencia repetitiva. Chatman nos ofrece una primera aproximación al concepto punto de vista muy válida:

En el uso ordinario se puede distinguir al menos tres significados:

a) literal: a través de los ojos (percepción) de alguien;

b) figurativo: a través de la visión del mundo de alguien (ideología, sistema conceptual, Weltanschauung, etc.);

c) transferido: desde la posición de interés de alguien (caracterizando su interés general, provecho, bienestar, salud, etc.) (1990, p. 163)

En el relato audiovisual, desde el momento en que se selecciona una posición de cámara y se elige un objetivo fotográfico con su correspondiente ángulo de visión ya se esta estableciendo de punto de vista básico que configurará la imagen en su totalidad, mostrando un espacio con unos personajes y elementos del decorado y dejando otros personajes y elementos del decorado en fuera de campo. Pero también determina la manera en que esos personajes serán mostrados. Como señala Zunzunegui

Toda imagen expresa inevitablemente un punto de vista, cargándose de intencionalidad, de juicio sobre lo que se muestra, ya que la mera operación de recortar

DOI: ri14.v11i1.192 | ISSN: 1697-8293 | Año 2013 Volumen 11 Nº1 | ICONO14 
un encuadre en el continuum de lo visible, debe ser leída como operación reveladora de una voluntad o si se quiere, más precisamente, de un sentido. (1998, p. 187)

El encuadre ya establece una primera selección que implica un punto de vista de forma que condiciona la percepción del espectador. Cuando se utiliza la frecuencia repetitiva con el objetivo de transmitir distinta información al espectador en cada ocasión, el encuadre de cada una de las repeticiones suele ser diferente de forma que al mostrar tanto un contenido novedoso en la segunda ocasión como una nueva manera de exponer el acontecimiento, los sucesos diegéticos adquieran una nueva dimensión y un nuevo sentido para el espectador.

Es muy frecuente, como ya se ha indicado, que la repetición se articule a través del relato de un narrador intradiegético. La presencia del narrador sirve tanto para justificar la frecuencia narrativa mediante procedimientos intradiegéticos como para acreditar el nuevo punto de vista, es decir, la nueva información que se supone procede de alguien que de algún modo ha podido vivir el acontecimiento. La forma en que se cuenta un acontecimiento resulta vital para su interpretación y para dotarle de veracidad. Ricoeur señala que "el término historia, en la mayor parte de las lenguas europeas, presenta una curiosa ambigüedad, pues significa, al mismo tiempo, lo que ha sucedido realmente y el relato de esos acontecimientos" (1999, p. 133). Transmitir un relato a partir de un narrador intradiegético permite justificar diegéticamente la frecuencia repetitiva y dotar al relato de verosimilitud. El procedimiento básico consiste en exhibir una parte de la historia con graves carencias informativa que no permiten al espectador entender en toda su amplitud lo sucedido, ni tampoco atribuir determinados hechos a ningún personaje concreto.2 A través de la presencia de un narrador intradiegético, que vuelve a relatar los acontecimientos ya mostrados, se potencia el mecanismo de credibilidad que tiene lo relatado. Es ahora cuando parece que la verdad va a aflorar frente a una información anterior incompleta. Pero conviene realizar algunas consideraciones. Así, para Francisco García

El narrador además de tener información de los hechos, tiene también creencias sobre las cosas y sobre los propios hechos que tiene que referir, por tanto es difícil dilucidar qué es realmente lo que corresponde a lo puramente referencial y qué es lo que se debe a sus creencias. (2011, p. 18)

El narrador expone desde una perspectiva distante en el tiempo algo que ocurrió $\mathrm{y}$, por lo tanto, es susceptible de realizar todo tipo de matices, además de poder 
incluir su opinión particular sobre los acontecimientos que aunque puedan estar afectándole todavía, ya han tenido lugar. Al respecto señala Chatman

Pero lo que el narrador relata desde su perspectiva está casi siempre fuera de la historia (heterodiegético), aunque sólo sea retrospectivo, es decir, distante temporalmente. Generalmente está recordando su propia percepción anterior como personaje. Pero ese recuerdo es una concepción, ya no es una percepción. (1990, p. 166)

El narrador intradiegético, cuando ejerce como tal y articula su relato en flashback, ya conoce el desarrollo global del acontecimiento y los efectos que tuvo. Pero hay algo más: el narrador deviene en un convencionalismo narrativo en numerosas ocasiones. Es habitual que a través del narrador se proceda a relatar acontecimientos que muy difícilmente pueden tener justificación en su saber. Y esto es debido a que la figura del narrador esta subordinada a las necesidades informativas del espectador y al adecuado desarrollo informativo. Para Sánchez Noriega

En rigor, la perspectiva o punto de observación desde un personaje implica que el fragmento del relato sólo narra los hechos conocidos por el narrador. Sin embargo, tanto en la novela como en el cine, se permite incluir hechos conocidos posteriormente, sabidos a través de otro, deducidos por la evolución de los acontecimientos, etc. Tanta es la libertad en este sentido que, con frecuencia, se llega a violar toda lógica, como sucede, por ejemplo, en La vida es bella (La vita è bella, Roberto Benigni, 1998), donde el relato se presenta como un largo flashback introducido y clausurado por la voz en off del personaje del niño, desde cuyo punto de vista se narraría toda la historia, cuando es evidente que ni los acontecimientos anteriores a su nacimiento son conocidos por él con tantos detalles ni, sobre todo, lo son conocidos con la perspectiva humorística que tiene la narración (2000, pp. 93-94)

Llegados a este punto, conviene establecer una diferencia entre el punto de vista y la voz narrativa, tal y como hace Chatman el punto de vista es el lugar físico o la situación ideológica u orientación concreta de la vida con los que tienen relación los sucesos narrativos. La voz, por el contrario, se refiere al habla o a los otros medios explícitos por medio de los cuales se comunican los sucesos y los existentes al público. El punto de vista no es la expresión, sólo es la perspectiva con respecto a la que se realiza la expresión. La perspectiva y la expresión no tienen por qué coincidir en la misma persona. (1990, p. 164)

DOI: ri14.v11i1.192| ISSN: 1697-8293 | Año 2013 Volumen 11 Nº1 | ICONO14 
Todavía debemos añadir una cuestión muy importante al concepto de punto de vista y narrador: hay que considerar que en el relato audiovisual el saber y la visión no tienen que concordar. En otras palabras, el narrador y la cámara no siempre coinciden en una misma imagen: un narrador puede estar contando algo y la imagen puede mostrar un punto de vista que nunca pudo tener como personaje y que no se le puede atribuir (Gómez Tarín, 2011, p. 56). Algunas teorías que toman como referencia exclusivamente el hecho literario, tienden a confundir ambos aspectos. Pero en la narrativa audiovisual el saber y el mirar son aspectos muy diferentes que no se deben equiparar.

La película española El extraño viaje nos ofrece un magnífico ejemplo sobre la utilización combinada de la frecuencia repetitiva y la alteración del punto de vista entre secuencias para crear primero expectativas en el espectador y posteriormente resolverlas. El análisis será completado con otros dos ejemplos más concretos tomados de Ciudad de Dios y El hombre que mató a Liberty Valance donde se parte del mismo principio pero con matices diferentes. En todos los ejemplo analizados el acontecimiento es siempre el mismo en las dos apariciones en el discurso, no experimenta ninguna modificación sustancial, no hay ningún cambio en su desarrollo diegético, sino que el diferente punto de vista condiciona la información que recibe el espectador. Diferente es el caso de otros relatos, como por ejemplo Rashomon, donde cada repetición, en este caso asociada al relato de diferentes narradores, presenta acontecimientos sustancialmente alterados. Inclusive en el ejemplo de Rashomon podemos decir hay un tipo de frecuencia repetitiva muy diferente, ya que en sentido estricto la cadena de acontecimientos no es similar en cada una de las repeticiones en el discurso.

\section{Frecuencia repetitiva y variación del punto de}

\section{vista en El extraño viaje}

El extraño viaje es una película española del año 1964, dirigida por Fernando Fernán Gómez y con guion de Pedro Beltrán. Los sucesos se desarrollan en un pueblo que parece situado próximo a Madrid. La película se puede dividir en dos partes. En la primera parte hay dos líneas argumentales básicas. Una línea argumental 
trata sobre la vida de tres hermanos: Ignacia, Paquita y Venancio. Se muestran una serie de acontecimientos que finalizan con la muerte de los tres hermanos, primero de Ignacia a manos de su hermano Venancio y posteriormente los otros dos hermanos, Paquita y Venancio. Estas últimas muertes plantean un enigma, ya que no se sabe cómo murieron y si alguien les mató, aunque se supone que un tercer personaje misterioso fue el asesino. Otra línea argumental trata sobre las relaciones entre Fernando, un músico que acude los fines de semana al pueblo para tocar, y Beatriz que trabaja en la paquetería del pueblo. En esta primera parte no aparece ningún narrador diegético. La segunda parte se articula principalmente a partir de un largo flashback de Fernando uno de los personajes protagonistas y que se convierte en narrador diegético. El flashback se divide en nueve fragmentos y presenta las siguientes características:

1. Repite acontecimientos que se vuelen a mostrar desde otro punto de vista. En la mayor parte de las repeticiones, el nuevo punto de vista introduce elementos novedosos que permiten entender el acontecimiento mostrado anteriormente con otro sentido o completar la información de la primera vez. Es por lo tanto un caso claro de frecuencia repetitiva con modificación del punto de vista.

2. A partir del flashback se explican sucesos ocurridos en la primera parte sin necesidad de volver a mostrarlos, sino que se recurre a mostrar acontecimientos por primera vez. En este caso no hay frecuencia repetitiva.

Centrándonos en la frecuencia repetitiva, hay que considerar que en El extraño viaje se repite bien la misma acción básica en sentido estricto o bien una situación compuesta por varias acciones como, por ejemplo, la acción que se desarrolla en la plaza del pueblo, un baile, y simultáneamente la acción que tiene lugar en casa de los hermanos, si bien predomina la repetición de una acción básica.

En la primera parte de la película a través del control de la información se genera un enigma, una expectativa sobre lo qué está ocurriendo realmente y sobre quién es el responsable de los sucesos. Este es un procedimiento narrativo muy conocido

En general, el argumento conforma nuestra percepción de la historia controlando 1) la cantidad de información de la historia a la que tenemos acceso; 2 ) el grado de pertinencia que podemos atribuir a la información presentada; y 3) la correspondencia formal entre la presentación del argumento y los datos de la historia. (Bordwell, 1999, p.54)

DOI: ri14.v11i1.192| ISSN: 1697-8293 | Año 2013 Volumen 11 Nº1 | ICONO14 
Los sucesos claves que hacen referencia a los hermanos, especialmente los relativos a las muertes, son presentados de forma que generen un enigma: "Sólo aplazando la revelación de alguna información puede el argumento crear anticipación, curiosidad, suspense y sorpresa." (Bordwell, 1999, p. 55). El enigma o la duda se establece en la primera vez que se muestra el acontecimiento por dos procedimiento narrativos básicos, uno temporal la elipsis y otro espacial, el fuera de campo. A través de la elipsis se elimina un fragmento de la historia clave para comprender el verdadero sentido de los sucesos o para evitar mostrar un personaje. Tal y como señala Sánchez-Escalonilla: “Dentro de las elipsis estratégicas se encuentran las paralipsis, consistentes en la omisión de un dato o momento, y que vienen a ser la clave de la resolución de un guion." (2001, p. 165).

La elipsis provoca una falta significativa de información que a su vez causa un enigma.

El fuera de campo plantea otros matices. El espacio diegético es siempre el resultado del espacio en campo (aquello que es mostrado con nitidez) y el espacio fuera de campo (aquello que no se muestra). Un plano al mismo tiempo que muestra algo, deja algo sin mostrar. Pero los elementos presentes en campo remiten a un espacio fuera de campo. Espacio en campo y fuera de campo son completamente dependientes entre sí. Para Gómez Tarín

La relación entre campo y fuera de campo es biunívoca, no puede existir el uno sin el otro [...] Lo presente (lo visto en la imagen) se encuadra en un conjunto que, a su vez, hemos de subsumir en un conjunto superior, con su hipotético campo y fuera de campo, y así sucesivamente hacia el infinito hasta formar un todo homogéneo que es el universo de lo representado, pero no podemos entenderlo como un todo cierto, puesto que forma parte del imaginario; ese Todo no es sino lo Abierto, que remite al tiempo e incluso al espíritu antes que al espacio y la materia (formado, como está, por la "materia" de los sueños) . (2011, p. 260)

Para Gómez Tarín la procedencia y vigencia del fuera de campo es exclusivamente cinematográfica, audiovisual, a diferencia de la elipsis que tiene una relación con los procedimientos narrativos literarios.

A continuación, vamos a analizar el flashback de Fernando y la frecuencia repetitiva para comprobar como condiciona la información que recibe el espectador y estructura el relato. 
Los cuatro primeros fragmentos del flashback no vuelven a mostrar ningún acontecimiento y sirven para aclarar al espectador la relación entre Fernando e Ignacia, relación a la que no se hace ninguna referencia en la primera parte de la película. Su función principal es, por lo tanto, completar la primera parte de la historia. Es un flashback externo, es decir, que cuenta acontecimientos que ocurrieron antes del primer acontecimiento con el que se inicia el relato y que sirve para establecer el presente del relato o relato primero en la terminología de Genette (1989). Los acontecimientos y situaciones que se repiten son analizados a continuación tanto la primera vez que aparecen como la segunda vez en el relato de Fernando, agrupados por unidad de espacio y tiempo.

1. Plano subjetivo de un personaje al que no se muestra en el interior de una casa. Por el movimiento de cámara suponemos que es la visión de un personaje, ya que como señala Jost (1995, p. 141): "un plano, o bien está anclado en la mirada de una instancia interna a la diégesis y se produce entonces una ocularización interna, o bien no conlleva una tal mirada y es una ocularización cero". Se dirige hacia una habitación y comienza a abrir una puerta. En la repetición se muestra a Fernando dentro de la casa. Es muy significativo que en el relato sin narrador tengamos un plano subjetivo del personaje Fernando y en el relato del propio Fernando no. Como ya hemos comentado, la cámara y el relato del narrador no tienen necesariamente que coincidir. La acción prosigue en la primera vez que se muestra el acontecimiento con un corte a otra puerta que se abre. Es Paquita que va a la habitación de su hermano y lo despierta para decirle que ha oído ruidos, como si alguien estuviera en la casa. Ambos se dirigen a la habitación de Ignacia. Se ve luz por debajo de la puerta de la habitación de Ignacia. Venancio toca a la puerta y la llama. Ignacia abre la puerta. No se puede ver el interior de la habitación ya que Ignacia se sitúa en la puerta y oculta en espacio interior. Mientras hablan, tiene lugar un relámpago que hace que Ignacia se gire y se pueda vislumbrar a alguien dentro de la habitación. Paquita lo ve y pregunta: “¿Quién hay ahí, Ignacia?” Ignacia niega la presencia de alguien en su habitación y les ordena a sus hermanos que se marchen. Ignacia entra en la habitación. Los hermanos dan media vuelta pero en vez de marcharse vuelven hacia la habitación de Ignacia. Paquita escucha a través de la puerta lo que ocurre dentro. Se pone muy nerviosa y Venancio tiene que taparle la boca: se acaba de enterar que Ignacia quiere deshacerse de ellos. Debe quedar muy claro que en ningún momento se sabe quién está en la habitación con 
Ignacia. En la repetición, se observa a Fernando dentro de la habitación de Ignacia y a los hermanos fuera. Como se puede comprobar es la misma acción expuesta desde otro punto de vista: desde dentro de la habitación de Ignacia. Ahora sabemos quién estaba en esa habitación. Hay por lo tanto repetición. La secuencia prosigue mostrando a Ignacia que logra deshacerse de sus hermanos y le dice a Fernando que piensa librarse de ellos. Sabemos, porque ya se ha visto anteriormente la misma acción, que Paquita esta fuera de la habitación escuchando y se entera de los propósitos de su hermana Ignacia. En todo este fragmento hay repetición y al mostrar el mismo acontecimiento desde otro punto de vista la información queda restablecida en su totalidad. Es necesario observar los dos puntos de vista para entender adecuadamente lo que ocurre en el universo diegético propuesto por el relato. Hay que tener en cuenta que los dos puntos de vista se pueden ir alternando (lo más habitual) de forma que solamente sea necesario mostrar una vez el acontecimiento para que todo quede claro. Obviamente, en este procedimiento no se crea un enigma. En la película El extraño viaje se opta por dejar en suspense determinados acontecimientos para posteriormente volver sobre ellos y resolver el enigma.

2. Se muestra la habitación de Venancio de noche. Se escucha el sonido de una puerta. Los hermanos van a la habitación de Ignacia y en su ausencia entran. En la repetición a través del flashback se muestra a Ignacia acompañando a Fernando a la salida. Por este motivo no estaba Ignacia en su habitación. En la primera vez que se muestran los acontecimientos, vuelve Ignacia y les sorprende. Se muestra una serie de acontecimientos menores que afectan a Fernando y los músicos, pero que sirven para situar al espectador, y se regresa a la habitación de Ignacia con los hermanos. Suena en off la música de la orquesta. Ignacia, muy enfadada por la intromisión de sus hermanos, golpea a Paquita y Venancio mata a Ignacia golpeándola con una botella de aguardiente. Se alternan planos del baile y de la habitación, hasta que finalmente, Paquita le dice a Venancio que Ignacia está muerta. Se escucha en off la puerta que se abre, los hermanos miran en esa dirección, la cámara panea hacia la puerta. La puerta se abre lentamente. Se corta a un plano de los hermanos mirando a la puerta y gritan. Se utiliza tanto el fuera de campo, para evitar mostrar al personaje que entra por la puerta, como la elipsis para omitir lo que ocurre a continuación. Seguidamente se muestra un plano de las piernas de unos personajes que trasladan el cuerpo de Ignacia por unas escaleras. Plano de las piernas en la bodega llevando a Ignacia y a continuación tirándola en una cuba. El

ICONO14 | Año 2013 Volumen 11 № 01 | ISSN: 1697-8293 | DOI: ri14.v11i1.192 
uso del espacio fuera de campo nos impide ver a los personajes e identificarlos. Hay claramente un enigma sobre las causas del grito de los hermanos y sobre el misterioso personaje que ayuda a deshacerse del cuerpo de Ignacia. ¿Cómo se repite este fragmento esencial para entender cómo se desarrollaron los acontecimientos? Se muestra a Fernando entrando en la habitación de Ignacia. Grito de los hermanos. La cámara hace una panorámica y muestra a los hermanos y a Ignacia en el suelo. Fernando comprueba que está muerta. En este caso hay repetición en la entrada de Fernando con respecto a la primera ocasión en que se muestra. La continuidad de la acción (Fernando con los hermanos) está eliminada en la primera parte. Después se muestra como los hermanos y Fernando llevan a Ignacia a la bodega y la arrojan en un tonel. Nuevamente tenemos repetición de la acción, pero en este caso mostrando a los personajes que arrojan a Ignacia. El enigma respecto a Ignacia ha quedado resuelto, principalmente a partir de sucesos que se han vuelto a mostrar desde otro punto de vista restituyendo el espacio fuera de campo y ofreciendo la imagen completa que fue omitida en la primera vez.

3. Se muestra a Fernando en Madrid despidiéndose de sus compañeros músicos. Hermanos en la habitación de Ignacia. Realizan diversas acciones (comen, registran la habitación, etc.) hasta que se disponen a brindar con unas copas de pipermín. Se va la luz y se abre la puerta de repente entrando un personaje al que no se le puede identificar, los gatos saltan despavoridos de la cama y los hermanos gritan cayendo desmayados. La repetición de este bloque comienza mostrando a Fernando con sus compañeros músicos. Se despide de ellos. Esta repetición tiene una función clara de enlace con los sucesos siguientes y sitúa al espectador en un momento concreto de la trama. Continuación, Fernando vuelve a casa de Ignacia y se viste con las ropas de la difunta. Entra en la habitación donde están sus hermanos que gritan despavoridos: han confundido a Fernando con Ignacia. Esta parte es una repetición desde otro punto de vista y sirve para explicar y justificar posteriores acontecimientos diegéticos que tienen como protagonista a Fernando disfrazado de Ignacia.

4. Se muestra a unos abuelos de tertulia en la plaza y consumiendo unos vinos. Un abuelo señala al balcón y dice que Ignacia está tomando un aperitivo. La cámara panea a la izquierda y se observa la silueta de dos personajes en el balcón. El espectador sabe perfectamente que no puede ser Ignacia ya que está muerta y ha sido arrojada a un tonel de vino. Se insiste en el enigma del personaje miste-

DOI: ri14.v11i1.192 | ISSN: 1697-8293 | Año 2013 Volumen 11 Nº1 | ICONO14 
rioso. Llega Beatriz intercambia unas palabras con los abuelos y se marcha. En la repetición, Fernando disfrazado de Ignacia está tomando el aperitivo en el balcón. Una vez más tenemos una repetición desde otro punto de vista lo que nos permite completar la información y dar a los acontecimientos un nuevo significado. En esta película queda claro que dependiendo del punto de vista los acontecimientos tienen un sentido u otro. Se observa desde el balcón a los abuelos con Beatriz y a Beatriz que se marcha caminando.

5. Los hermanos y un tercer personaje se marchan del pueblo. Un vecino les observa. Este acontecimiento es el último que se vuelve a repetir tal cual.

La primera parte de la película finaliza con los cuerpos de los dos hermanos muertos en la playa, una mujer los observa y grita. Un nuevo enigma que se desvelará más adelante a través del flashback de Fernando, donde se narra como los tres llegan a la playa. Una vez allí tienen que esperar a una motora. Cuando comienza a aparecer, los hermanos se aceran a la orilla, Fernando se queda solo y aprovecha para poner un somnífero en las copas de Venancio y Paquita. Fernando les lleva las copas. Los tres bridan y se muestra a los hermanos bebiendo el champán con el veneno. Aquí no se repite el acontecimiento, sino que se muestra por primera vez la forma en que Fernando mató a los hermanos.

$\mathrm{Si}$ analizamos con cuidado las repeticiones, nos daremos cuenta de que todas son esenciales para entender el desarrollo de la historia; podríamos decir que resuelven todas las dudas planteadas en la primera parte. En buena medida, lo que se hace es restituir el espacio mostrado en fuera de campo en la primera parte mediante el cambio de punto de vista o retomar una acción para continuar mostrando lo que ocurrió y que en la primera parte se omitió por el uso de la elipsis. También hay que insistir en que se crea un enigma en la primera parte que se resuelve en la segunda por el uso de la frecuencia repetitiva cambiando el punto de vista.

\section{Frecuencia repetitiva y variación del punto de vista en El hombre que mató a Liberty Valance}

Hay otros ejemplos donde también se utiliza la frecuencia repetitiva y la variación del punto de vista para dar información sobre acontecimientos diegéticos 
fundamentales en la historia de ambos relatos, pero con matices muy diferentes al uso que hemos observado en El extraño viaje.

Un caso ejemplar lo encontramos en la película El hombre que mató a Liberty Valance, dirigida por Ford en 1962. Se parte de un dato falso que no se cuestiona hasta que finalmente se descubre lo que en realidad ocurrió. Se muestra en dos ocasiones un acontecimiento que tuvo lugar una única vez en la historia: la muerte del bandido Liberty Valance. Cada vez que se muestra, es un personaje distinto el responsable de su defunción. Obviamente, es a través de la repetición la manera de mostrar al personaje que realmente mató a Liberty Valance.

Nos encontramos con una planificación muy diferente en cada una de las ocasiones en que se muestran el mismo acontecimiento: la muerte del bandido Liberty Valance. La primera vez que se muestra el suceso tenemos una secuencia muy montada basada en el plano-contraplano de los dos personajes principales de la acción: Liberty y Stoddard. La planificación contribuye de manera importante a destacar la importancia del acontecimiento a partir del duelo de los dos protagonistas, aislándolos del resto del espacio y resaltando el dramatismo de la contienda. Desde el momento en que sale Liberty del salón la planificación es la siguiente:

1. PG. Sale Liberty del salón, situado al fondo. En primer término a la izquierda se encuentra Ransom Stoddard dispuesto a enfrentarse al bandido. Todos los planos generales de la primera vez que se muestra la muerte de Liberty tendrán un punto de vista muy similar a este primer plano general.

2. PA de Stoddard junto a otros dos personajes. Avanza hacia el lugar donde está Liberty hasta casi salir de campo.

3. PML de Liberty.

4. PG Se van aproximando los dos hombres.

5. PML de Liberty.

6. PE de Stoddard.

7. PML de Liberty.

8. PA de Stoddard avanzando.

9. PML de Liberty que desenfunda y dispara.

10. PA de Stoddard. El disparo destroza un botijo pero no alcanza a Stoddard.

11. PML de Liberty riéndose.

12. PA de Stoddard.

13. PM de Liberty.

DOI: ri14.v11i1.192| ISSN: 1697-8293 | Año 2013 Volumen 11 Nº1 | ICONO14 
14. PML de Stoddard.

15. PM de Liberty que vuelve a disparar.

16. PM de Stoddard que suelta el arma al ser alcanzado en el brazo por el disparo de Liberty.

17. PM de Liberty riendo.

18. PM de Stoddard.

19. PM de Liberty.

20. PG de ambos. Stoddard intenta recoger el revolver y Liberty dispara.

21. PD del revolver.

22. PG de ambos. Stoddard recoge el revolver con la mano del brazo sano y se dirige a Liberty.

23. PML de Liberty que apunta con cuidado.

24. PGC. Ambos personajes disparan. Liberty cae herido de muerte, se levanta y vuelve a caer. La cámara lo sigue en una suave panorámica. Sale la gente del salón. Todo parece indicar que Stoddard ha sido capaz de matar a Liberty.

Queda claro que es una secuencia con muchos planos, muy montada, con constante plano-contraplano de los protagonistas. De un total de 24 planos, hay un plano detalle, cinco planos generales y 18 planos de los protagonistas de los que ocho son de Stoddard y diez de Liberty. Pero es muy importante destacar que hay también, y a pesar del número de planos, un espacio fuera de campo que nunca es mostrado: el espacio frontal al edificio en cuyos bajos se desarrolla el duelo. Es un espacio que pasa desapercibido y que parece no tener importancia, ya que en ningún momento hay una referencia que lo destaque.

La segunda vez que se muestra el acontecimiento se hace con una planificación muy distinta e introduciendo planos desde un punto de vista distinto que permiten tener una visión muy diferente de lo que en realidad ocurrió. Los planos son los siguientes:

1. PG frontal al edificio donde se esta desarrollando el duelo en el exterior. Es un nuevo punto de vista. La espalda del personaje Tom Doniphon cubre el plano que está en negro, pero avanza y entra en campo permitiendo que se vea la imagen. Doniphon se sitúa a la izquierda. Liberty dispara, Stoddard deja caer el revolver y se agacha para recogerlo. Se está repitiendo una acción ya mostrada desde un nuevo punto de vista. 
2. PMC de Tom Doniphon observando la escena.

3. PG. Stoddard recoge el revolver.

4. PMC de Tom Doniphon.

5. PG. Panorámica de cámara a la derecha. Pompey arroja un rifle a Tom Doniphon que lo carga. Liberty apunta y también Doniphon a Liberty. Todos disparan. Liberty cae muerto. Doniphon arroja el rifle a Pompey y sale de campo cubriendo con su cuerpo el ángulo de cámara hasta que la imagen queda en negro.

En esta repetición, queda claro que Stoddard no mató a Liberty, sino que fue Tom Doniphon. Es a través de un plano general tomado desde otra posición como se muestra la verdad de los acontecimientos. Pero no es el plano general (también había plano general en la primera vez que se mostro el duelo), sino la perspectiva, el diferente punto de vista lo que marca la diferencia informativa. En la primera ocasión hay un espacio fuera de campo que nunca se muestra y resulta, lo vemos en la repetición, que es precisamente ahí donde se desarrolla el suceso clave. En este ejemplo no hay enigma, no hay ninguna duda en la primera vez que se muestra el suceso, sino un objetivo muy claro que consiste en que el espectador llegue a una conclusión falsa a partir de una información deficiente. Para conseguir este efecto, obviamente, el espectador no debe saber que tiene una información parcial. Es un caso muy diferente al de El extraño viaje, donde sí que se crea un enigma que posteriormente será resuelto. Por lo tanto, el procedimiento narrativo también es diferente.

\section{Frecuencia repetitiva y variación del punto de}

\section{vista en Ciudad de Dios}

En la película Ciudad de Dios dirigida por Fernando Meirelles y Katia Lund en 2002 se utiliza también la frecuencia repetitiva. Hay dos ejemplos que vamos a analizar para comprobar el uso diferente de la frecuencia repetitiva. En la primera modalidad, el procedimiento narrativo consiste en que la primera vez que se muestra el suceso se produce una elipsis de forma que se interrumpe su normal desarrollo y no se muestra una parte significativa. De esta forma, se plantea una incógnita sobre algún aspecto del acontecimiento, lo que justificará que más adelante se

DOI: ri14.v11i1.192| ISSN: 1697-8293 | Año 2013 Volumen 11 Nº1 | ICONO14 
vuelva a repetir una parte de la acción que sirve como enlace (con lo que tenemos frecuencia repetitiva) para proseguir mostrando el resto del acontecimiento y completar la información que recibe el espectador para dotar de pleno sentido a la situación diegética. Este es el procedimiento utilizado en Ciudad de Dios para explicar la desaparición de Marreco, hermano de uno de los protagonistas. En la primer ocasión se muestra a Marreco huyendo. En su fuga se encuentra con dos pequeños delincuentes, Dadinho y Bené, a los que les quita el dinero. A Marreco nadie lo vuelve a ver. Más adelante, cuando se explique la historia de Dadinho, se vuelve a mostrar el momento en que Marreco se encuentra con los pequeños delincuentes (frecuencia repetitiva) y se prosigue con la acción: Dadinho mata a Marreco. Ya sabemos el motivo de que nadie más volviera a ver a Marreco. El fragmento de acción repetida sirve como enlace para retomar el suceso.

En la segunda modalidad, el acontecimiento se vuelve a mostrar integro pero desde una nueva perspectiva, tal y como ya hemos visto. Pero en este caso no se genera ni un enigma para resolverlo más tarde ni sirve para ofrecer datos falsos al espectador y posteriormente ofrecer la versión cierta. La frecuencia repetitiva se utiliza para explicar un acontecimiento desconcertante. Hacia el final de la película tiene lugar un tiroteo entre las dos bandas de delincuentes que controlan Ciudad de Dios. En un momento determinado, un niño es gravemente herido y Mané Galinha intenta ayudarle. Cuando Galinha se gira el niño le dispara por la espalda y lo mata. En principio este acontecimiento parece que no tiene sentido. Pero al repetir por segunda vez un atraco que cometió Galinha nos enteramos de los motivos: el niño es el hijo del vigilante al que mató Galinha. La primera vez que se mostró el acontecimiento, dentro de un sumario donde se muestran tres atracos, toda la atención recayó sobre la transformación que estaba experimentando Galinha que de salvar a una persona en el primer atraco que realiza pasa a matar a un vigilante en el tercer atraco. Pero ahora, en la repetición, el nuevo punto de vista permite que el espectador se centre en el niño y en el vigilante asesinado por Galinha. A través de la repetición de un acontecimiento ya mostrado, se explica un nuevo suceso.

ICONO14 | Año 2013 Volumen 11 № 01 | ISSN: 1697-8293 | DOI: ri14.v11i1.192 


\section{Conclusiones}

A modo de conclusión, las características básicas del uso conjunto de la frecuencia repetitiva y de la variación del punto de vista se pueden resumir en las siguientes:

1. El uso conjunto de la frecuencia repetitiva y la alternancia del punto de vista suele usarse para dar un nuevo sentido a un suceso previamente mostrado. El acontecimiento es siempre el mismo en las dos o más apariciones en el discurso, no se produce ninguna transformación sustancial, sino que al modificar el punto de vista se condiciona la información que recibe el espectador. Una serie de ejemplos muy obvios los hemos analizado en la película El extraño viaje donde la utilización de la frecuencia repetitiva y la modificación del punto de vista entre secuencias permiten crear expectativas y posteriormente resolverlas. Un caso muy claro de lo que estamos comentando es la supuesta (e imposible) presencia de Ignacia una vez que ha sido asesinada en el balcón. En la repetición se descubre que estaba siendo suplantada por otro personaje.

2. El sentido final se adquiere gracias a la repetición, ya que en la primera ocasión hay una falta real de información para entender adecuadamente un suceso, ya sea el acontecimiento que se repite u otro. En El extraño viaje hay un ejemplo que aclara lo que estamos comentando: el misterioso personaje que se encuentra en la habitación de Ignacia al principio de la película. Posteriormente, a través del relato de Fernando convertido en narrador delegado, se descubre su identidad.

3. Una modalidad muy frecuente consiste en plantear un enigma o duda sobre algún acontecimiento o situación diegética. La repetición del acontecimiento servirá para resolver la incógnita planteada. Es el caso de la muerte de los hermanos (Paquita y Venancio) en El extraño viaje. Una vez más, a través de la repetición de la situación diegética se resuelve el enigma.

4. La frecuencia repetitiva con variación del punto de vista también se utiliza para dar una nueva información que modifique el sentido adquirido en la primera vez que se ha mostrado el acontecimiento, como es el caso analizado de El hombre que mató a Liberty Valance. En este caso no hay ningún tipo de enigma. Sin embargo, al repetir el acontecimiento se establece que no fue Stoddard el autor de la muerte del bandido Liberty, sino Tom Doniphon. A través de la repetición se muestra al personaje que realmente mató a Liberty Valance.

DOI: ri14.v11i1.192| ISSN: 1697-8293 | Año 2013 Volumen 11 Nº1 | ICONO14 
5. Un acontecimiento que se muestra por primera vez en el relato también se puede explicar repitiendo un suceso ya mostrado pero variando el punto de vista para que consiga un nuevo significado. Por ejemplo, la muerte de Mane Galinha por los disparos de un niño solamente adquiere sentido cuando se vuelve a mostrar un atraco donde se observa a Mane Galinha asesinando al padre del niño que le dispara.

6. Los recursos fundamentales para lograr una reserva informativa en la primera ocasión en que se muestra el acontecimiento son la elipsis, como es el caso de la desaparición de Marreco en la película Ciudad de Dios, y el fuera de campo, como en la película El hombre que mató a Liberty Valance. Cuando se muestra por segunda vez el acontecimiento se restituye la información restringida en la primera ocasión.

\section{Referencias}

Bal, M. (1990). Teoría de la narrativa. Madrid: Cátedra

Bordwell, D. (1999). La narración en el cine de ficción. Barcelona: Paidós

Chatman, S. (1990). Historia y discurso. Madrid: Taurus.

García, F. \& Rajas, M. (Ed.). (2011). Narrativas Audiovisuales: el relato. Madrid: Icono14.

Gaudreault, A. \& y Jost, F.(1995). El relato cinematográfico. Barcelona: Paidós

Genette, G. (1989). Figuras III. Barcelona: Lumen.

Gómez Tarín, F.J.(2011). Elementos de narrativa audiovisual. Expresión y narración. Santander: Shangrila ediciones.

Kohan, S.A. (2005). El tiempo de la narración. Barcelona: Alba.

Ricoeur, P. (1999). Historia y narratividad. Barcelona: Paidós.

Sánchez Noriega, J.L. (2000). De la literatura al cine. Barcelona: Paidós

Sánchez-Escalonilla, A. (2001). Estrategias de guion cinematográfico. Barcelona: Ariel

Zunzunegui, S. (1998). Pensar la imagen. Madrid: Cátedra 